\title{
Paramagnetic Meissner Effect and Strong Time Dependence at High Fields in Melt-Textured High- $T_{C}$ Superconductors
}

\author{
Cristol de Paiva GouvêA* \\ Divisão de Metrologia de Materiais, Instituto Nacional de Metrologia, \\ Qualidade e Tecnologia (INMETRO), Duque de Caxias 25250-020, Brazil \\ Fábio Teixeira Dias, Valdemar das Neves Vieira and Douglas Langie da Silva \\ Departamento de Física, Universidade Federal de Pelotas, Pelotas 96010-900, Brazil \\ Jacob SCHAF \\ Instituto de Física, Universidade Federal do Rio Grande do Sul, Porto Alegre 91501-970, Brazil \\ Frederik WOLFF-FABRIS \\ Dresden High Magnetic Field Laboratory, Helmholtz-Zentrum Dresden-Rossendorf, 01314 Dresden, Germany \\ Joan Josep Roa RovirA \\ Université de Poitiers, Poitiers 86962, France
}

(Received 31 May 2012)

\begin{abstract}
In this work we report on systematic field-cooled magnetization experiments in melt-textured $\mathrm{YBa}_{2} \mathrm{Cu}_{3} \mathrm{O}_{7-\delta}$ samples containing Y211 precipitates. Magnetic fields up to $14 \mathrm{~T}$ were applied either parallel or perpendicular to the ab planes and a strong paramagnetic response related to the superconducting state was observed. This effect is known as paramagnetic Meissner effect (PME). The magnitude of the PME increases when the field is augmented. This effect shows a strong paramagnetic relaxation, such that the paramagnetic moment increases as a function of the time. The pinning by the Y211 particles plays a crucial role in the explanation of this effect and our results suggest that the pinning capacity can produce a strong flux compression into the sample, originating the PME and the strong time dependence.
\end{abstract}

PACS numbers: 74.81.Bd, 74.25.Wx, 74.72.-h

Keywords: Melt-textured YBaCuO, Paramagnetic Meissner effect, Time dependence

DOI: $10.3938 / \mathrm{jkps} .62 .1414$

\section{INTRODUCTION}

The magnetic properties of the high temperature superconductors have been widely studied by different research groups around the world since their discovery. In relation to the magnetic properties, the Meissner effect is one important signature of the superconductivity. In this case, a diamagnetic moment is observed due to exclusion of the magnetic flux of the interior of the superconducting sample when the temperature is below the critical temperature $\left(T_{C}\right)$. This important property allows to distinguish a superconducting material from a conducting one, but in several cases the superconducting materials exhibit a paramagnetic response instead of the conventional diamagnetism. This effect is sometimes

*E-mail: cristol_@hotmail.com called paramagnetic Meissner effect or PME. In this case, the magnetic flux is not expelled, and a paramagnetic state can be generated.

This effect is observed in several magnetic field regimes, and in some cases the paramagnetic moments increases with the applied magnetic field, but in others the paramagnetic response decreases when the magnetic field is increased. The PME was first observed in polycrystalline samples of high temperature superconductors [1] and the discovery of this effect has originated an enormous effort of investigation by several groups and several theories and models have been proposed to explain this interesting and controversial behavior. One of the first interpretations is based on the occurrence of Josephson $\pi$ junctions between superconducting grains into the sample, and in this case the superconducting samples showing the PME can be modeled as a Josephson medium 


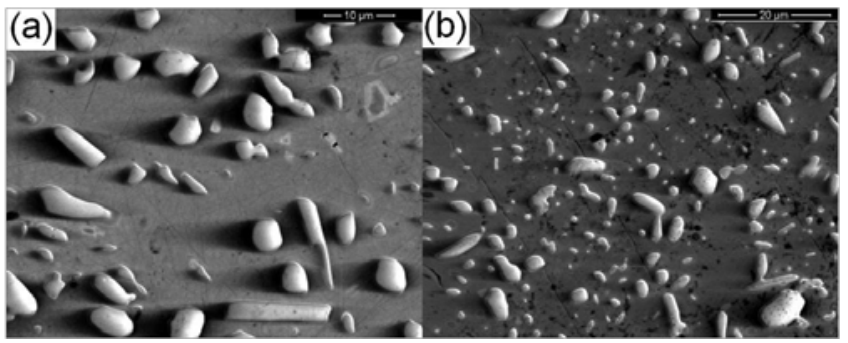

Fig. 1. SEM images obtained from Bridgman (a) and topseeding (b) samples indicating the presence of Y211 particles.

where the $\pi$ junctions are randomly distributed [2,3]. By another way, some experimental results have motivated the proposal of mechanisms based on flux trapping and a strong flux compression into the superconducting material, and a non equilibrium compressed flux states can be stabilized by inhomogeneously cooling the sample, and a paramagnetic moment can be originated [4]. The formation of a giant vortex state on the sample, or the flux capture by samples with a particular shape, may produce a strong compression of the trapped flux into the sample $[5,6]$.

Other models based on arrays of multiply-connected superconductors have been proposed $[7,8]$. In this case Josephson junctions are taken into account and a paramagnetic moment appears when inner currents may flow through these junctions under specific conditions.

An interesting and important result observed in superconducting samples that exhibit the PME is the anomalous time-dependence on the magnetization $[9,10]$. In some cases, the paramagnetic moments obtained after field cooling process (FC procedure) relax monotonically to increasingly positive values. Some results show that this tendency remains unaltered for large time intervals. In other cases, the relaxation may show a change of sign in the magnetization from negative to positive during the same experiment. This behavior is opposite to expectations based on a flux-creep scenario, yielding this anomalous effect more curious and instigating.

\section{EXPERIMENTS AND DISCUSSION}

Two different melt-textured $\mathrm{YBa}_{2} \mathrm{Cu}_{3} \mathrm{O}_{7-\delta}$ (YBCO) samples were investigated. The samples were grown by Bridgman (Bridgman sample) and top-seeding techniques (top-seeding sample). The similar description of the preparation process may be found in Refs. 11 and 12. The samples after the growth present a directional structure with a fine distribution of precipitates of the $\mathrm{Y}_{2} \mathrm{BaCuO}_{5}(\mathrm{Y} 211)$ phase, added during the growth. The purpose of the addition of the Y211 phase is to introduce a considerable number of pinning centers. This procedure enhances strongly the flux pinning potential in melt-textured YBCO samples. Figure 1 shows typ-

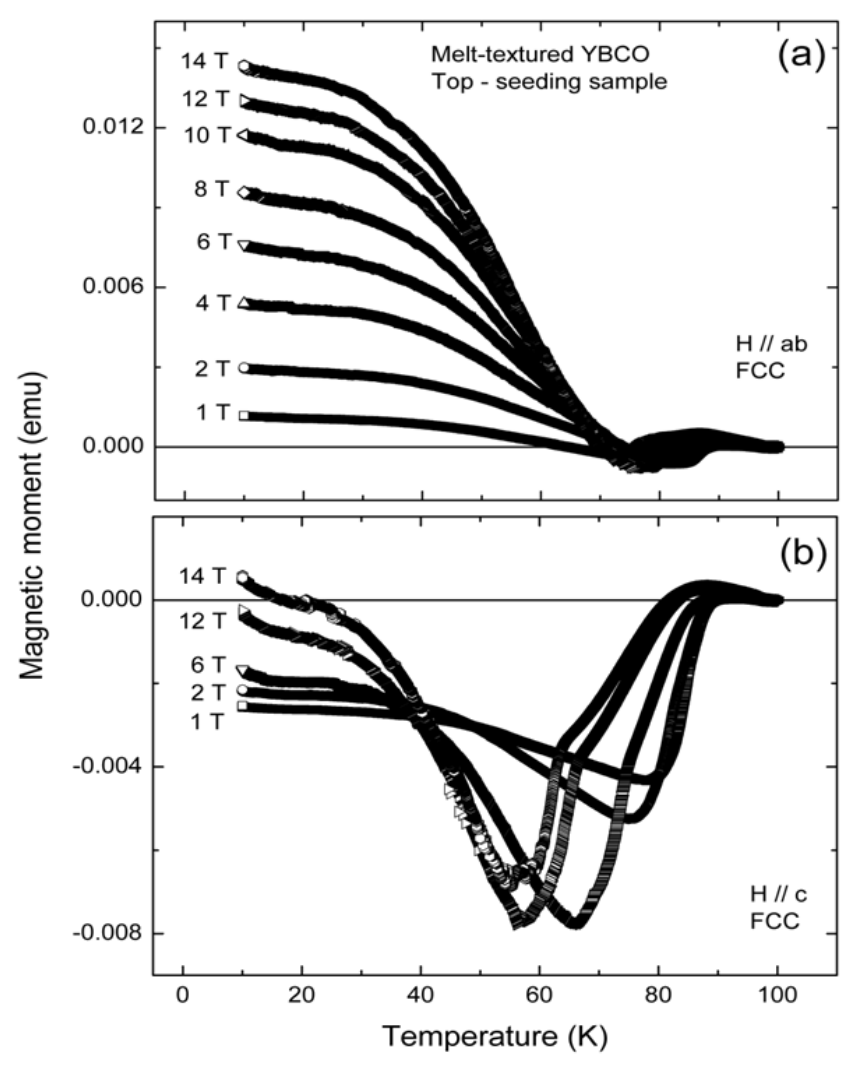

Fig. 2. FCC experiments performed at top-seeding sample when the magnetic field is applied parallel (a) and perpendicular (b) to the $a b$ plane. The paramagnetic moments increase monotonically with the magnetic applied field, mainly when the magnetic field is applied along the $a b$ plane. The magnetic contribution of the Y211 phase is subtracted.

ical SEM images obtained from Bridgman (Fig. 1(a)) and top-seeding (Fig. 1(b)) samples, indicating the presence of Y211 particles dispersed into the superconducting matrix. The measured samples were cut out from the melt-textured samples into the form of small and crystallographically oriented parallelepipeds. Typical sample dimensions are $0.66 \times 0.88 \times 2.68 \mathrm{~mm}^{3}$, with the largest dimension oriented parallel to the c-axis. Magnetization measurements up to $14 \mathrm{~T}$ were performed using a SQUID magnetometer by Quantum Design and a magnetic property measurement system also by Quantum Design.

Magnetic moments were measured as functions of the temperature according to zero-field cooling (ZFC) and field-cooled cooling (FCC) procedures. The time dependence of the FCC moment at fixed temperature and magnetic field was also measured. All results were corrected for the corresponding demagnetizing factors and the magnetic contribution of Y211 particles were removed.

Figure 2 shows FCC experiments performed at topseeding sample when the magnetic field is applied parallel (Fig. 2(a)) and perpendicular (Fig. 2(b)) to the ab plane. These results show that the magnitude of the 

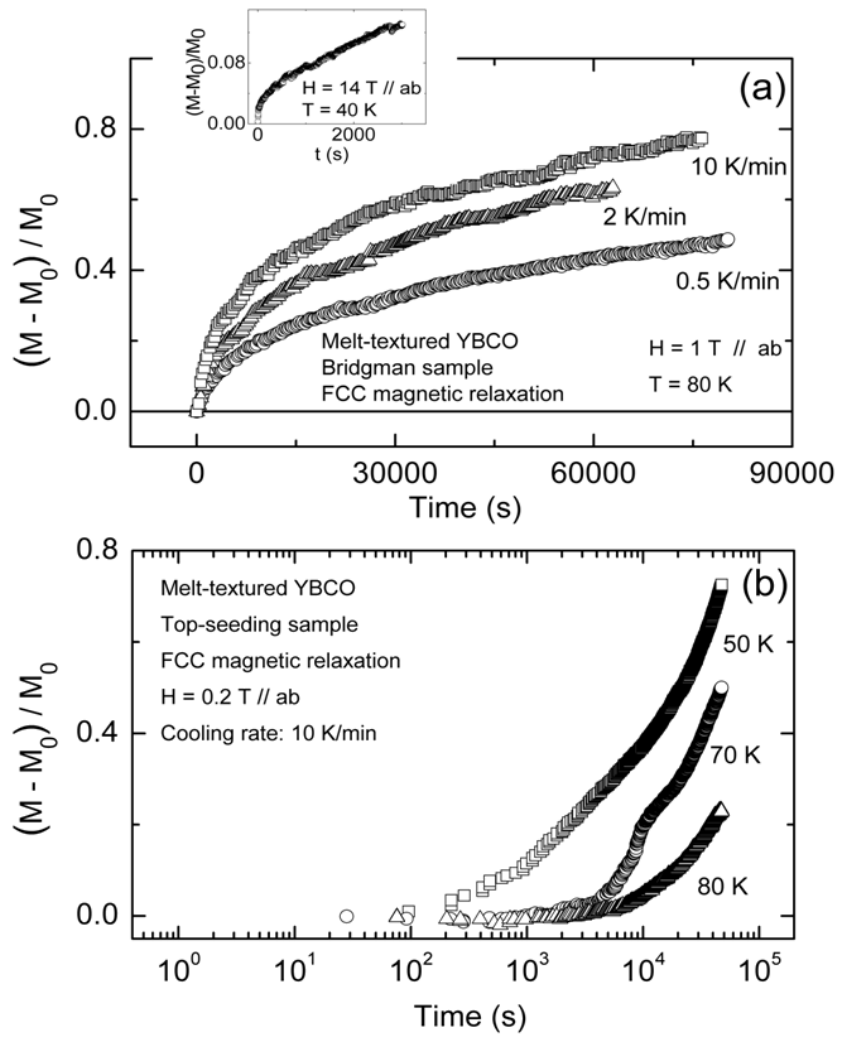

Fig. 3. Time dependence of the normalized FCC moments for the top-seeding and Bridgman samples. (a) The magnitude of the paramagnetic moments depend on the cooling rate. The inset shows that the paramagnetic relaxation can occurs in magnetic fields as high as $14 \mathrm{~T}$. (b) A nonlogarithmic time dependence is observed for different temperatures for the top-seeding sample. The measurements are performed at $\mathrm{H}=2 \mathrm{kOe}$ applied parallel to the $a \mathrm{~b}$ plane and for a fixed cooling rate of $10 \mathrm{~K} / \mathrm{min} . \mathrm{M}_{0}$ is the initial magnetic moment.

paramagnetic moment increases monotonically with the magnetic applied field, even when the spin contribution from the Y211 particles were removed from the raw data. The Fig. 2(a) shows a diamagnetic dip below $T_{C}$, but the magnetic moment increases steadily as the temperature is lowered, showing a slight tendency to saturate. By another hand, when the magnetic field is applied along the c-axis, the paramagnetic effect shows an anisotropic characteristic, as can be seen in the Fig. 2(b). In this case the magnetic moment is positive only for the higher magnetic field and for the lower temperatures. The experimental results presented in the Fig. 2 constitute the first observation of the PME in this field range in $\mathrm{YBCO}$ samples.

Figure 3 shows the time dependence of normalized FCC magnetization for the Bridgman (Fig. 3(a)) and top-seeding (Fig. 3(b)) samples. The Fig. 3(a) shows the effect of different cooling rates for Bridgman sample. In the Fig. 3(a) the measurements were done after cooling the sample in rates of $0.5,2$ and $10 \mathrm{~K} / \mathrm{min}$. It's possible to observe that the magnitude of the FCC moment depends crucially on the cooling rate of the sample. Similar results were observed in the top-seeding sample. The inset in the Fig. 3(a) shows that the magnetic moment relaxes in magnetic fields as high as $14 \mathrm{~T}$. The Fig. 3 (b) shows results obtained from the top-seeding sample. The measurements were performed at $\mathrm{H}=2 \mathrm{kOe}$, cooling rate of $10 \mathrm{~K} / \mathrm{min}$, and different temperatures. Its possible to observe that the magnetic moment shows a strong deviation from the logarithmic time dependence. Similar results were also observed in the Bridgman sample.

Our results can be explained in terms of a magnetic flux compression scenario modulated by a strong pinning effect due to Y211 particles. In essence, the effect observed in our samples is caused by the inhomogeneous cooling of the sample below the superconducting transition, as shown by the sensitivity on the cooling rate. In a previous work Koshelev and Larkin [4] showed that in an inhomogeneous cooling the magnetic flux near the edges of the sample can be expelled towards its interior, originating a compressed flux scenario stabilized by pinning during the superconducting state. The SEM images of both samples corroborate to this scenario. The flux compression into the sample can be so strong that in some regions the vortex density is reduced below the expected from equilibrium state, opening place for the admission of extra vortices, originating paramagnetic moments. This admission of extra vortices evolves with the time, producing a paramagnetic relaxation observed in both investigated samples.

\section{CONCLUSION}

In conclusion, we have presented results on the FCC magnetization experiments in two melt-textured YBCO samples containing Y211 precipitates. We have observed strong paramagnetic moments during the FCC experiments. The paramagnetic moments increase monotonically with the magnetic applied field up to $14 \mathrm{~T}$. This is the first observation of this effect in this field range. The effect also shows an anomalous and curious time dependence, such that the paramagnetic moment increases as a function of time. Our results can be explained by a model based on a flux compression scenario into the sample modulated by strong pinning due to Y211 particles dispersed in the superconducting matrix.

\section{ACKNOWLEDGMENTS}

This work was financed by the Brazilian Ministry of Science and Technology and State of Rio Grande do Sul, under the Grant PRONEX FAPERGS/CNPq 10/0009-2, the Ministry of Science and Technology, under the Grant CAPES/Procad 059/2007, and the PRONEM 03/2011 program, under contract $\mathrm{N}^{\circ} 11 / 2042-7$. 


\section{REFERENCES}

[1] P. Svedlindh et al., Phys. C 162/164, 1365 (1989).

[2] M. Sigrist and T. Rice, Rev. Mod. Phys 67, 503 (1995).

[3] W. Braunish et al., Phys. Rev. Lett. 68, 1908 (1992).

[4] A. Koshelev and A. Larkin, Phys Rev. B 52, 13559 (1995).

[5] V. Moshchalkov, X. Qiu and V. Bruyndoncx, Phys. Rev. B 55, 11793 (1997).
[6] A. Geim et al., Nature 396, 144 (1998).

[7] P. Barbara et al., Phys. Rev. B 60, 7489 (2000).

[8] A. Nielsen et al., Phys. Rev. B 62, 14380 (2000).

[9] F. Dias et al., Phys. Rev. B 70, 224519-1 (2004).

[10] A. Terentiev et al., Phys. Rev. B 60, R761 (1999).

[11] X. Obradors et al., Phys. Rev. B 50, 7032 (1994).

[12] X. Obradors et al., Supercond. Sci. Technol. 10, 884 (1997). 